\title{
Causes of Positive Pretransplant Crossmatches in the Absence of Donor-Specific Anti-Human Leukocyte Antigen Antibodies: A Single-Center Experience
}

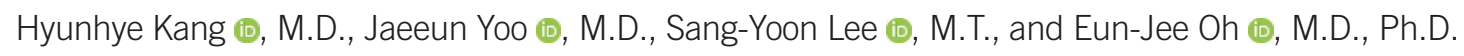 \\ Department of Laboratory Medicine, Seoul St. Mary's Hospital, College of Medicine, The Catholic University of Korea, Seoul, Korea
}

Pretransplant crossmatch (XM) testing is widely used for detecting preformed donor-specific antibodies (DSAs) against human leukocyte antigen (HLA). However, in some cases, there is a positive XM result in the absence of HLA-DSAs, the cause of which was rarely identified. We reviewed the causes of sequential positive XM results at a single center and analyzed the presence of non-HLA antibodies in patients with an unexplained positive pretransplant XM result. Among 251 patients with T-cell/B-cell complement-dependent cytotoxicity (CDC) or flow cytometric crossmatch (FCXM) positivity, HLA-DSAs were confirmed in 88 (35.1\%) by a single antigen bead (SAB) assay, 150 (59.8\%) used rituximab (anti-CD20), and 13 (5.2\%) had neither HLA-DSAs nor a desensitization history. Anti-angiotensin II type 1 receptor IgG and 33 non-HLA antibodies were tested in the 13 patients with an unexplained positive pretransplant XM result, and more than one non-HLA antibody were revealed in all these patients; 11 patients had non-HLA antibodies reported to be associated with graft rejection, and two patients experienced rejection episode after kidney transplantation. Our study suggests considering non-HLA antibodies testing when a CDC or FCXM test is positive without a definite cause. Assessing non-HLA antibodies might be useful for interpreting XM results and evaluating immunologic risk in transplant recipients.

Key Words: Complement-dependent cytotoxicity, Flow cytometric crossmatch, Donor-specific antibodies against human leukocyte antigen, non-HLA antibodies, Transplantation
Received: May 14, 2020

Revision received: July 20,2020

Accepted: November 24, 2020

Corresponding author:

Eun-Jee Oh, M.D., Ph.D.

Department of Laboratory Medicine, Seoul St. Mary's Hospital, College of Medicine,

The Catholic University of Korea, 222

Banpo-daero, Seocho-gu, Seoul 06591,

Korea

Tel: +82-2-2258-1641

Fax: +82-2-2258-1719

E-mail: ejoh@catholic.ac.kr

\section{(c) (i) (8)}

(c) Korean Society for Laboratory Medicine

This is an Open Access article distributed under the terms of the Creative Commons Attribution Non-Commercial License (https://creativecommons.org/licenses/by-nc/4.0) which permits unrestricted non-commercial use, distribution, and reproduction in any medium, provided the original work is properly cited.
Cell-based physical XM testing, including complement-dependent cytotoxicity (CDC) and flow cytometric crossmatch (FCXM), remain the fundamental techniques for predicting the risk of rejection prior to organ transplantation. The main cause of a positive XM result is the presence of preformed antibodies that recognize and react specifically to donor HLA molecules [1, 2]. However, there are other factors that cause positive XM result in the absence of donor-specific antibodies (DSAs). HLA-DSAs are detected through a sensitive single-antigen bead (SAB) assay; however, other low-level DSAs, allele-specific antibodies, HLADSAs from recent allosensitization, and antibodies against HLA antigens not contained in the assay beads can be missed by this test. The therapeutic antibody rituximab, which is frequently used for desensitization prior to organ transplantation, can also lead to positive B-cell XM results in the absence of HLA-DSAs [3]. In addition, autoantibodies and non-HLA antibodies can cause a positive XM result [4]. The presence of non-HLA antibodies, such as antibodies against angiotensin II type I receptor (AT1R), has been reported to produce a positive FCXM result and has been associated with allograft failure in transplant patients [5-8]. As non-HLA antibody-mediated rejections have been increasingly reported, the importance of non-HLA anti- 
body detection in pretransplant XM testing has been recently emphasized [5, 9-13].

Despite the occurrence of positive XM results in the absence of HLA-DSAs and the fact that possible causes of this occurrence can only be explained for some patients, transplant laboratories rarely evaluate the presence of non-HLA antibodies in pretransplant XM test samples. We reviewed the causes of sequential positive XM results at a single center and analyzed the presence of non-HLA antibodies in patients with an unexplained positive pretransplant XM result.

This study was carried out from February 2020 to May 2020, and a total of 1,638 XM tests conducted in Seoul St. Mary's Hospital, Seoul, Korea, between January 2017 and February 2020 were enrolled. The XM results and clinical features of patients were determined by review of electronic medical records. Among them, 371 tests (22.6\%) showed a positive result in one or more XM tests, including T-cell/B-cell CDC and FCXM. If multiple XM tests were performed for the same donor and patient pair during the observation period, the first positive result was included for analysis. Exclusion criteria were repeated test results from a single donor-recipient pair $(\mathrm{N}=51)$, patients without donor HLA typing results ( $\mathrm{N}=64)$, and patients with only FCXM or CDC results $(\mathrm{N}=5)$. Finally, we focused on 251 patients with positive XM results. The Institutional Review Board of Seoul St. Mary's Hospital approved this study (KC2ORISI0186).

The XM testing conducted in our laboratory is as described previously [14]. In brief, total lymphocytes, and T and B lymphocytes were respectively isolated from $20 \mathrm{~mL}$ of donor fresh whole blood, using a cell isolation kit (EasySep, STEMCELLTechnologies, Vancouver, Canada). For the CDC test, the donor lymphocyte suspension was incubated with recipient serum followed by addition of the rabbit complement (One lambda, Canoga Park, CA, USA). The T-cell anti-human globulin antibody (AHG) test involved the addition of $1 \mu \mathrm{L}$ of $\mathrm{AHG}$ after incubation of donor cells with recipient serum. For the FCXM test, CD3PC5 and CD19-PC7 were used as markers to separate the T and $\mathrm{B}$ lymphocytes. All lymphocytes were treated with pronase (Type XIV, Sigma, St. Louis, MO, USA) (1.0 mg/mL) for 30 minutes at $37^{\circ} \mathrm{C}$ to minimize interference by the non-specific binding of human IgG to the Fc receptor on B lymphocytes. After incubation, lymphocytes were analyzed using a Navios flow cytometer (Beckman Coulter, San Diego, CA, USA). In cases where pronase-induced T-cell positive or IgM autoantibody-induced B-cell CDC-positive XM results were suspected, additional tests were performed using pronase-untreated or dithiothreitol-treated lymphocytes, respectively. The cutoff value for a positive T-cell (>1.17) and B-cell (>1.14) FCXM result was determined as the mean fluorescent intensity (MFI) ratio, with three standard deviations, obtained using HLA antibody-negative sera, as recommended [15].

In all positive XM patients, the LABScreen Single Antigen kit (One Lambda) was used to detect HLA antibodies. All patients and donors were typed for HLA-A, -B, -C, -DRB1, -DQA1, and -DQB1 using the LABType SSO kit (One Lambda). Ambiguities were resolved by sequence-based typing (SBT). SBT was performed using AlleleSEQR (GenDx, USA), and sequencing was performed on an 3500 Genetic Analyzer (Thermo fisher, USA). A positive DSA result was defined as having at least one HLADSA (HLA-A, B, Cw, DR, or DQ), with an MFI $\geq 1,000$ identified using the SAB assay. All DSAs were confirmed by eplet analysis, using the HLA matchmaker program, as previously described [16]. Positive DSA results are listed, along with their median values and 95\% confidential intervals, in Table 1. We classified the XM tests into CDC, AHG, and FCXM types and DSAs into Class I DSAs and Class II DSAs to identify patients in each subgroup. A chi-square test was performed to compare the clinical features between DSA positive and negative groups.

To determine whether the positive XM results were generated by non-HLA antibodies, we additionally tested for the presence of anti-AT1R IgG antibodies and 33 non-HLA antibodies in the 13 patients with an unexplained positive pretransplant $X M$ result using leftover sera from XM tests. Anti-AT1R IgG antibodies were detected using a commercially available ELISA kit (One Lambda), as previously described [7]. To determine the nonHLA antibody profile, LABScreen Autoantibody group 1 (32 targets) and group 2 (one target) kits (One Lambda) were further used, according to the manufacturer's instructions. In brief, 40 $\mu \mathrm{L}$ of serum and $10 \mu \mathrm{L}$ of autoantibody microsphere mix were incubated for 30 minutes in the dark at room temperature (20$24^{\circ} \mathrm{C}$ ). After washing, phycoerythrin-conjugated anti-human IgG was added and incubated for 30 minutes. After washing, the samples were analyzed on a LABScan3D flow analyzer (One Lambda). A positive non-HLA antibody reaction was defined by a value above the reference background value of each antigencoated bead provided by the manufacturer. The positive non$H L A$ antibody reaction values were obtained from the adjusted MFI values based on the 95th percentile values from non-transplanted individuals.

Desensitization therapy was performed for 225 (89.6\%) of 251 patients with pretransplant DSAs and/or positive XM results [17]. Briefly, rituximab, at a dose of $375 \mathrm{mg} / \mathrm{m}^{2}$ (Mabthera Genentech, Inc., San Francisco, CA, USA), was administered two 


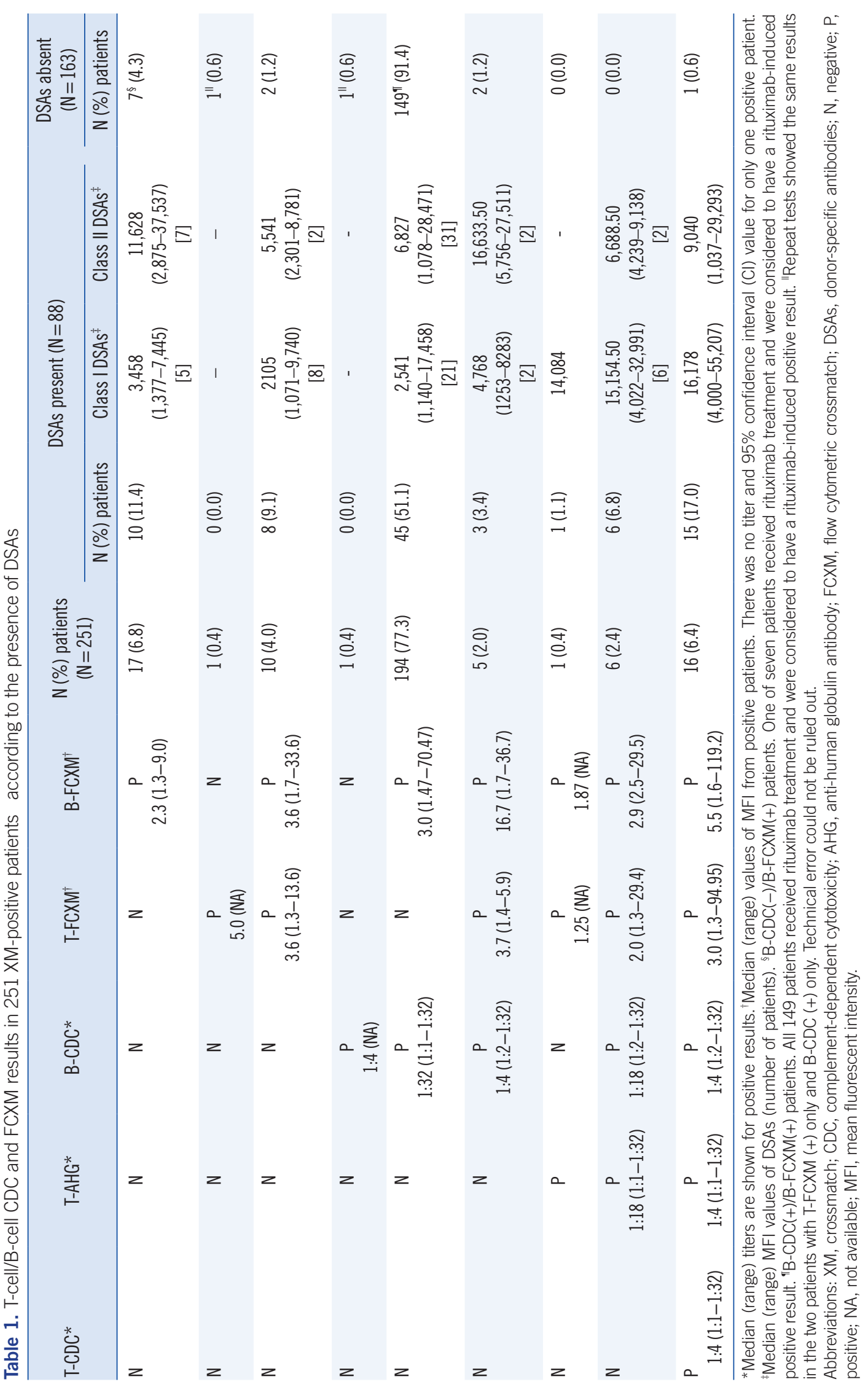


to three weeks prior to transplantation, and plasmapheresis/intravenous immunoglobulin (100 mg/kg) therapy was initiated 13 days before transplantation and administered every 48 hours.

Among the 251 patients with positive XM results, 88 (35.1\%) had DSAs against donor HLAs. Gender (females), sensitization (transfusion, pregnancy, and transplantation) history, and HLA antibody positivity were significantly associated with DSA positivity ( $P$ value $<0.05$ ). XM results of the T-cell/B-cell CDC and FCXM tests are summarized according to the presence of DSAs in Table 1. Among the 163 XM-positive/DSA-negative patients, the 150 that showed only positive B-cell XM result [149 B-cell CDC(+)/B-cell FCXM(+) and 1 B-cell CDC(-)/B-cell FCXM(+)] were considered to have positive results due to rituximab therapy. These 150 patients received rituximab therapy 0-6 months before they showed a positive B-cell XM result. Since the initial XM results of all 150 patients were negative before rituximab treatment, the possibility of positivity induced by rituximab was strongly suggested. For the two out of 163 patients with T-cells, FCXM (+) only, and B-cell CDC (+) only, repeat tests confirmed the results; however, the possibility of technical error cannot be ruled out.

The remaining 13/163 (5.2\%) positive XM patients had nei- ther DSAs detected nor a relevant desensitization history.

Interestingly, all 13 patients with XM-positive/DSA-negative results had non-HLA antibodies with varying profiles (Fig. 1). Although all patients had antibodies against regenerating islet-derived protein 3-alpha, we could not estimate a direct relationship between a specific non-HLA antibody and XM result. Therefore, the combined reactions of various non-HLA antibodies may have caused a positive XM result. The baseline characteristics, XM results and detected antibodies in the 13 abovementioned patients are shown in Table 2. In previous studies, 11 of 33 nonHLA antibodies were related to a negative long-term outcome of transplantation [5, 7, 9, 13, 18].

Six of these 13 patients received kidney transplantation (KT) and two of them experienced a rejection episode. Both these patients had pre-transplant non-HLA antibodies (patient 2: C-XC motif chemokine 11 and interferon (IFN)- $\gamma$; patient 7: vimentin, glutathione S-transferase theta-1, and peroxisomal trans-2enoyl-CoA reductase), which are associated with chronic renal allograft injuries or poor prognosis of transplantation [5, 9-13]. At the time of the rejection episode, patient 2 showed decreased MFI values for non-HLA antibodies, whereas patient 7 showed similar MFI values and developed de novo non-HLA antibodies

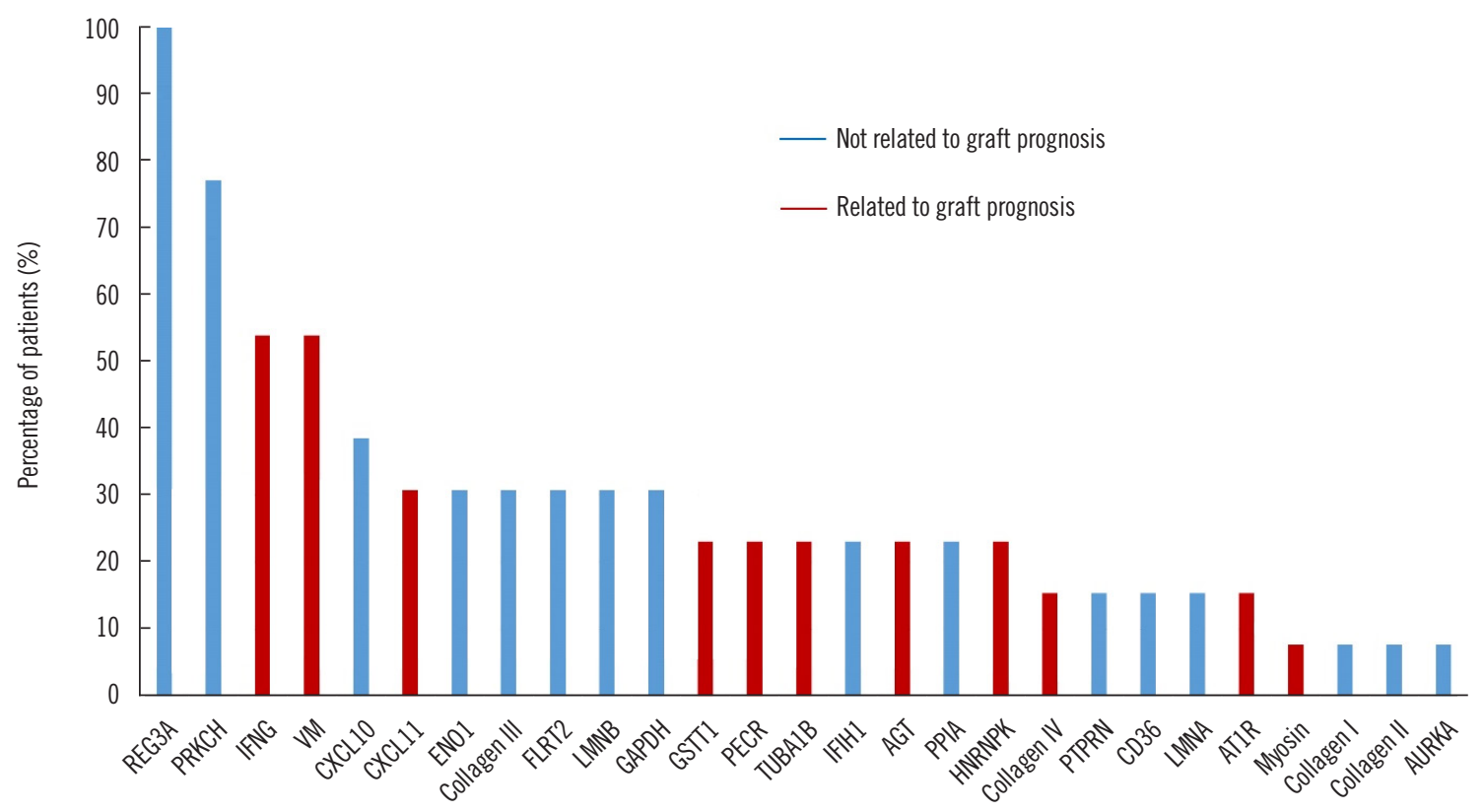

Fig. 1. Detection of non- HLA antibodies in 13 patients with positive XM results and an absence of DSAs.

Abbreviations: XM, crossmatch; DSAs, donor-specific antibodies; HLA, human leukocyte antigen; REG3A, regenerating islet-derived protein 3-alpha; PRKCH, protein kinase C eta type; IFNG, interferon gamma; VM, vimentin; CXCL10, C-X-C motif chemokine 10; CXCL11, C-X-C motif chemokine 11; ENO1, alphaenolase; FLRT2, leucine-rich repeat transmembrane protein; LMNB, lamin-B1; GAPDH, glyceraldehyde-3-phosphate dehydrogenase; GSTT1, glutathione Stransferase theta-1; PECR, peroxisomal trans-2-enoyl-CoA reductase; TUBA1B, tubulin alpha-1B chain; IFIH1, interferon-induced helicase $\mathrm{C}$ domain-containing protein 1; AGT, angiotensinogen; PPIA, peptidyl-prolyl cis-trans isomerase A; HNRNPK, heterogeneous nuclear ribonucleoprotein K; PTPRN, receptor-type tyrosine-protein phosphatase-like N; LMNA, prelamin-A/C; AT1R, angiotensin II type 1 receptor; AURKA, aurora kinase A-interacting protein. 
Kang $\mathrm{H}$, et al.

Positive pretransplant crossmatches with negative DSA

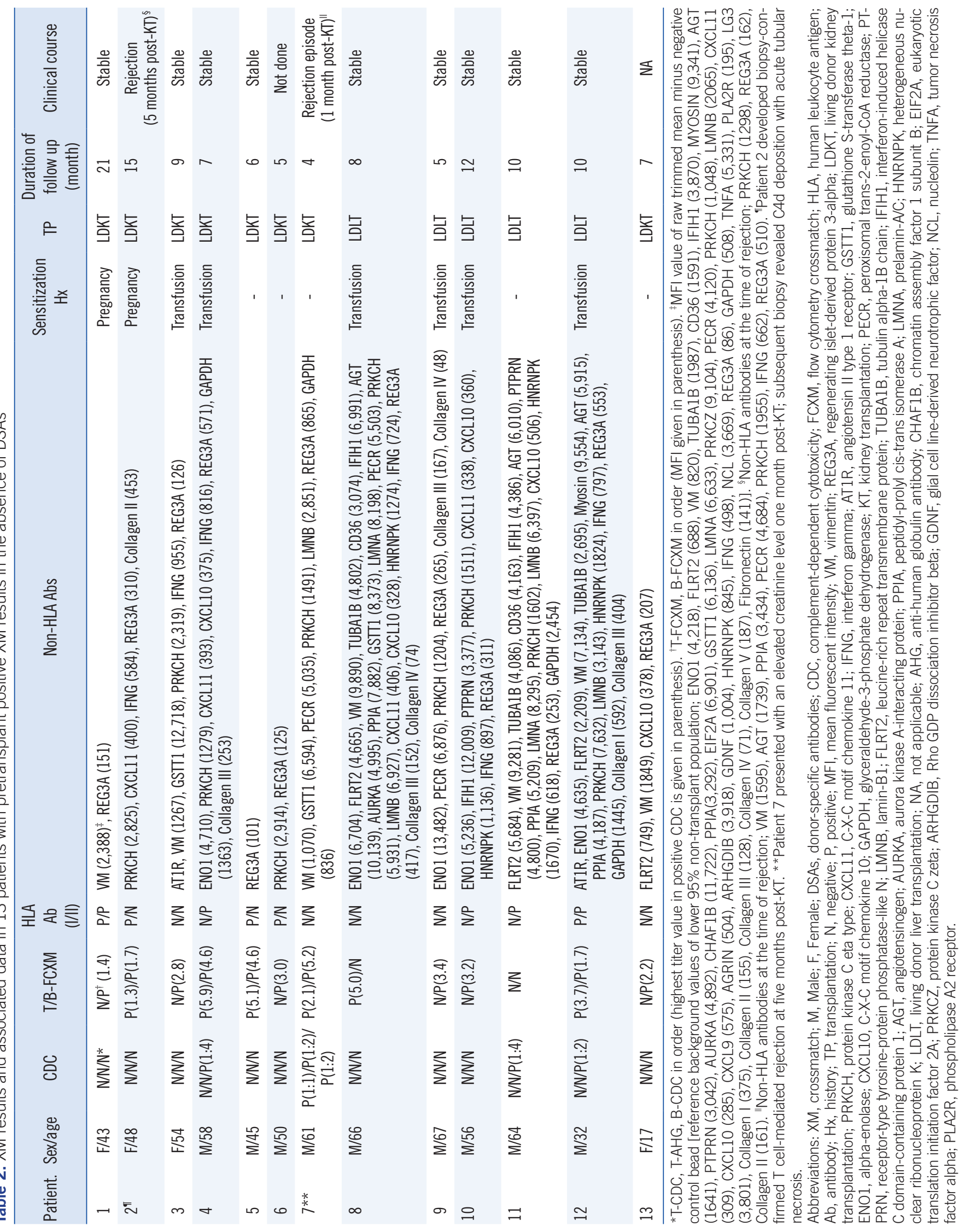


against angiotensinogen, peptidyl-prolyl cis-trans isomerase $\mathrm{A}$, and IFN- $\gamma$. The decreased MFI values may have been due to the absorption of non-HLA antibodies by the graft. Although non-HLA autoantibodies were identified in all unexplained positive pretransplant XM results, we could not find a direct relationship among the type or intensity of the autoantibodies, the MFI ratio of the positive XM result, and the graft outcome. These findings suggest that non-HLA antibodies interact with diverse factors to influence the clinical outcome.

This study has several limitations. As we did not perform HLADP typing for the donor, we could not analyze the possibility of a positive XM result caused by HLA-DP DSAs. Further studies focusing on DSAs against HLA-DP are needed. In addition, we could not confirm the presence of non-HLA antibodies after elution, and only a small number of patients were tested for nonHLA antibodies. Since we excluded patients who received rituximab therapy or were XM-negative from among those for whom non-HLA antibody tests were performed, we could not rule out the presence and impact of non-HLA antibodies in these patients. Nevertheless, we thoroughly analyzed all positive XM results recorded over a three-year period and found non-HLA antibody positivity in patients showing a positive XM result in the absence of HLA-DSAs. As the association between pretransplant non-HLA antibodies and development of HLA-specific antibodies has been established, non-HLA antibodies may indirectly lead to graft rejection [19].

In conclusion, we suggest considering a non-HLA antibody testing when a CDC or FCXM test result is positive without an identifiable cause. Assessment of non-HLA antibodies might further be useful for interpreting XM results and for evaluating the immunologic risk in transplant recipients. Further studies will be needed to elucidate the mechanism by which non-HLA antibodies are involved in the post-transplant immunologic reaction and their effect on the long-term prognosis.

\section{ACKNOWLEDGEMENTS}

None.

\section{AUTHOR CONTRIBUTIONS}

Oh EJ designed the study; Kang $\mathrm{H}$, Yoo J, and Lee SY contributed to acqusition of data; Oh EJ and Kang $\mathrm{H}$ analyzed and interpreted data; Kang $\mathrm{H}$ wrote the manuscript; Oh EJ edited the manuscript. All authors have read and agreed to the final manuscript.

\section{CONFLICTS OF INTEREST}

No potential conflicts of interest relevant to this article are reported.

\section{RESEARCH FUNDING}

This work was supported by a National Research Foundation of Korea grant funded by the Korean government (MSIP) (NRF2020R1A2B5B01001859).

\section{ORCID}

Hyunhye Kang

Jaeeun Yoo

Sang-Yoon Lee

Eun-Jee Oh https://orcid.org/0000-0003-1338-6451 https://orcid.org/0000-0001-9917-5393 https://orcid.org/0000-0002-1251-2877 https://orcid.org/0000-0001-5870-915X

\section{REFERENCES}

1. Mulley WR and Kanellis J. Understanding crossmatch testing in organ transplantation: A case-based guide for the general nephrologist. Nephrol (Carlton) 2011;16:125-33.

2. Kumar A, Mohiuddin A, Sharma A, El Kosi M, Halawa A. An update on crossmatch techniques in transplantation. J Kidney 2017;3:4.

3. Milongo D, Vieu G, Blavy S, Del Bello A, Sallusto F, Rostaing L, et al. Interference of therapeutic antibodies used in desensitization protocols on lymphocytotoxicity crossmatch results. Transpl Immunol 2015;32:151-5.

4. Park H, Lim Y, Han B, Hyun J, Song EY, Park M. Frequent false-positive reactions in pronase-treated T-cell flow cytometric cross-match tests. Transplant Proc 2012;44:87-90.

5. Zhang $Q$ and Reed EF. The importance of non-HLA antibodies in transplantation. Nat Rev Nephrol 2016;12:484-95.

6. Cardinal H, Dieudé M, Hébert MJ. The emerging importance of nonHLA autoantibodies in kidney transplant complications. J Am Soc Nephrol 2017;28:400-6.

7. Min JW, Lee H, Choi BS, Park CW, Yang CW, Kim YS, et al. Clinical impact of pre-transplant antibodies against angiotensin II Type I receptor and major histocompatibility complex class I-related chain A in kidney transplant patients. Ann Lab Med 2018;38:450-7.

8. Yu S, Huh HJ, Lee KW, Park JB, Kim S-J, Huh W, et al. Pre-transplant angiotensin II type 1 receptor antibodies and anti-endothelial cell antibodies predict graft function and allograft rejection in a low-risk kidney transplantation setting. Ann Lab Med 2020;40:398-408.

9. Aguilera I, Alvarez-Marquez A, Gentil MA, Fernandez-Alonso J, Fijo J, Saez C, et al. Anti-glutathione S-transferase T1 antibody-mediated rejection in C4d-positive renal allograft recipients. Nephrol Dial Transplant 2008;23:2393-8.

10. Dinavahi R, George A, Tretin A, Akalin E, Ames S, Bromberg JS, et al. Antibodies reactive to non-HLA antigens in transplant glomerulopathy. $J$ Am Soc Nephrol 2011;22:1168-78.

11. Li L, Sigdel T, Vitalone M, Lee SH, Sarwal M. Differential immunogenicity and clinical relevance of kidney compartment specific antigens after renal transplantation. J Proteome Res 2010;9:6715-21. 


\section{Kang $\mathbf{H}$, et al.}

12. Sigdel TK, Li L, Tran TQ, Khatri P, Naesens M, Sansanwal P, et al. NonHLA antibodies to immunogenic epitopes predict the evolution of chronic renal allograft injury. J Am Soc Nephrol 2012;23:750-63.

13. Sigdel TK and Sarwal MM. Moving beyond HLA: a review of $\mathrm{nHLA}$ antibodies in organ transplantation. Hum Immunol 2013;74:1486-90.

14. Lee H, Kim JI, Moon IS, Chung BH, Yang CW, Kim Y, et al. Investigation of serum angiotensin II type 1 receptor antibodies at the time of renal allograft rejection. Ann Lab Med 2015;35:314-20.

15. Hamrick $\mathrm{C}$ and Lebeck L. Flow cytometric $T$ and $B$ cell crossmatching. ASHI laboratory manual, 4th Ed. Philadelphia: American Society for Histocompatibility and Immunogenetics 2000:41-5.

16. Kim HS, Choi A-R, Yang M, Oh E-J. EDTA Treatment for Overcoming the Prozone Effect and for Predicting Clq Binding in HLA Antibody
Testing. Ann lab Med 2019;39:572-6.

17. Chung BH, Choi BS, Oh EJ, Park CW, Kim JI, Moon IS, et al. Clinical impact of the baseline donor-specific anti-human leukocyte antigen antibody measured by Luminex single antigen assay in living donor kidney transplant recipients after desensitization therapy. Transpl Int 2014;27:49-59.

18. Angaswamy N, Klein C, Tiriveedhi V, Gaut J, Anwar S, Rossi A, et al. Immune responses to collagen-IV and fibronectin in renal transplant recipients with transplant glomerulopathy. Am J Transplant 2014;14:68593.

19. Zhang $X$ and Reinsmoen NL. Impact of non-human leukocyte antigenspecific antibodies in kidney and heart transplantation. Front Immunol 2017;8:434. 